\title{
FONTAMARA DI SILONE: Il Verbo e la Parola
}

VinCENZO Di BonAVENTURA

Chi parla di vittorie? Resistere oggi è tutto.

RAINER MARIA RILKE

ABSTRACT: Con il presente articolo s'intende compiere un rapido exscursus sulla relazione tra le premesse pseudoreligiose dello scrittore Silone, il retroterra culturale cristiano nel quale nascono e vivono i personaggi della sua opera più celebre, e l'esito del suo percorso narrativo, divergente, se non antinomico, con tali premesse, e in virtù del quale il fine ultimo è nient'affatto coincidente con la causa prima. La ricerca, non certo consapevole, della parola da parte dei personaggi del romanzo determina la loro adesione alla terra e alla sua precarietà, e tale ricerca supera, anche qualitativamente, qualsiasi slancio mistico e morale dell'autore, per quanto corposo ed autentico esso sia, risolvendosi in una prosa avente il suo centro unicamente in se stessa. Attraverso il contributo di diversi testi critici, anche di vecchia data, l'intento è quello di mostrare come sia sempre esistita, in Italia come all'estero, una comune coscienza del valore artistico ed estetico di Fontamara, per quanto mai del tutto affermatasi sull'ancor oggi troppo conclamato messaggio politico e pedagogico, ma sempre capace di lasciar emergere, dal suo stato latente, una visione del mondo 
e della testimonianza umana nella quale l'implicito e la densità delle forme rappresentate sono la più grande, e forse unica, risorsa.

PAROLE CHIAVE: traduzione; dualismo; dialetto; viaggio; scrittura; Berardo Viola; testimonianza.

RESUMO: É intenção deste artigo fazer um breve excursus sobre as premissas pseudo-religiosas do escritor Silone e o êxito da sua narração que se revela divergente de tais premissas e, graças à contribuição de alguns textos críticos, mostrar como sempre existiu uma consciência do valor estético e artístico de Fontamara. Além da mensagem política, pedagógica ou religiosa, o que emerge é uma visão do mundo e do testemunho humano na qual a concretude da língua e a densidade das formas representadas são o maior e, talvez, o único recurso.

PALAVRAS-CHAVE: tradução; dualismo; dialeto; viagem; escritura; Berardo Viola; testemunho.

ABSTRACT: This article briefly surveys the relation between Silone's pseudo-religious premise, the Christian cultural background of Fontamara's characters, and the result of Silone's narrative trajectory, which completely diverges from any religious premise. The characters' search for a language determines their attachment to the land and its precarious nature, and this search qualitatively supersedes any mystical or moralistic idea that Silone might have had. The characters' search for a language results in a prose that has at its center only itself. Going through different critical texts, including older ones, my intention is to show how both in Italy and abroad there has existed a common conception of the artistic and aesthetic merit of Fontamara. Although the novel's political and pedagogical messages are still relevant today, Fontamara's greatest resources for the contemporary reader are to be found in its latent vision of the world, and in the form of the human testimony.

KEYWORDS: duality; translation; testimony; Berardo Viola; choralism; irony; human word. 


\section{I cafoni}

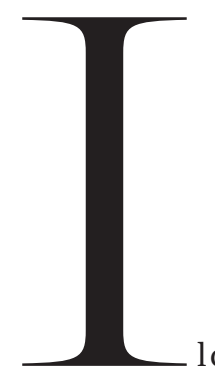

ldualismo tra la realtà e gli abitanti del piccolovillaggio montano prospiciente la piana del Fucino è il grande tema del romanzo Fontamara di Ignazio Silone.

L’antagonismo tra cafoni e cittadini è percepibile innanzitutto nell'antinomia linguistica tra le due parti. Silone sottolinea il fenomeno sin dalla prefazione del suo libro, paragonando la lingua italiana a una lingua morta o a una lingua straniera appresa a scuola senza alcun rapporto con il modo di agire, pensare ed esprimersi dei Fontamaresi. La lingua nazionale ha un carattere quasi coloniale, capace di esercitare una violenza sui contadini, distorcendo e corrompendo i loro pensieri e dando ad essi l'apparenza di una traduzione. Ma lo scrittore supera la dicotomia iniziale tra la comunità del borgo e il mondo esterno accettando i limiti che questa "traduzione" (Fontamara, p. 15, da cui si cita sempre) impone, affinché possa raccontare "la verità sui fatti di Fontamara" (p. 16). Così, improvvisamente, la traduzione non tradisce, ma connette. Tuttavia, se la lingua "è presa in prestito", la maniera di raccontare rimane per l'autore "un'arte fontamarese" (p. 16). La frattura tra città e borgo montano resta dunque insanabile e richiami a questa opposizione si presenteranno di continuo nei primi capitoli. La discussione che i cafoni hanno con il Cav. Pelino (ma si pensi anche all'episodio in cui la milizia fascista censisce politicamente gli uomini del borgo) è emblematica della loro incapacità di comprendere e spiegarsi e ciò che avvertono, essenzialmente, è la profonda ostilità della parola nei loro confronti. Con il suo linguaggio, intessuto di termini sconosciuti ai contadini, ${ }^{1}$ Pelino è il primo contatto con una neonata politica che, se da una parte mortifica il germe dell'espressione, dall'altra celebra l'eloquio più superfluo e magniloquente. 
Se parlare implica anche un fare, la loro inadeguatezza verbale è un tutt'uno con quella fisica. I contadini parlano e scrivono con la stessa goffaggine con cui si muovono e indossano in città le scarpe, il colletto e la cravatta. Si pensi, in tal senso, all'episodio della discesa delle donne al capoluogo. L'originalità dei cafoni sta nel presentarsi immediatamente, per quanto inconsapevolmente, come un elemento eversivo nei confronti del nuovo governo, fisicamente prima ancora che verbalmente.

Il fatto è che Silone considerava le parole la peggiore delle tirannie (SILONE, 1980), e in una conferenza romana ebbe a dire di avere "una tremenda paura della terribile ambiguità della parola” (SCARAMUCCI, 1980, p. 18). Pur servendosi dell'italiano e della traduzione, è implicita la condanna

di ogni possibilità che un qualunque sistema linguistico possa sostituirsi, per rappresentarlo nelle sue intrinseche espressioni, ad una altro sistema. La parola storpia il senso di un'altra parola (oltre che la parola stessa), la corrompe, dà soltanto l'apparenza di poterne penetrare l'originaria struttura. (DI GREGORIO, 1991, p. 17)

\section{La lingua}

L'autore compie un'operazione di mimesi diretta dell'idioma locale rintracciabile nelle apocopi sillabiche (Giuvà, Matalê), nella aferesi della sillaba iniziale di verbi come accecare (cecarmi, cecano), nell'uso di vari termini come zinale, scorze, cannella, e in generale attraverso la trasposizione di elementi dialettali e di costrutti della lingua popolare. Si pensi ad alcuni moduli fraseologici che ricalcano abitudini idiomatiche tipiche del meridione come l'accusativo retto da preposizioni (" $a$ voi nessuno vi ha invitato"), o il verbo andare al posto del verbo essere come reggente delle preposizioni soggettive ("Come va che non ha le corna e le zampe di caprone?"), o il per con valore di scopo (“Andare per elemosina”) o, ancora, la collocazione delle particelle pronominali proclitiche o enclitiche prima o dopo il verbo con valore semantico debole ("Berardo se ne va in pazzia"). I cognomi poi sembrano proprio la trasposizione di soprannomi originari (Cannarozzo, Cipolla, Ranocchia, Scamorza, etc...). ${ }^{2}$

Secondo Vittoriano Esposito, però, in Fontamara avviene un sostanziale rifiuto del dialetto. Moduli, sintagmi e stilemi di estrazione popolare ${ }^{3}$ sono scarsi e ciò determina, ad esempio, una certa differenza con gli abusi degli inserti dialettali tipici del neorealismo. In comune con il neorealismo l'autore avvertì l'urgenza di testimoniare, ma ne rifiutò essenzialmente l'ideologizzazione programmatica.

\footnotetext{
2. Si leggano, per un’analisi più dettagliata, le pagine 21-22 di P. SPEZZANI, «Fontamara» di Silone: grammatica e retorica del discorso popolare, Padova, Liviana, 1979, di cui l'inizio del presente paragrafo costituisce una breve sintesi.

3. Costruzioni come "Il mio stomaco non lo ritiene", "Bere a garganella", “...e noi gli avevamo sempre perdonato", o denominazioni come "Torsi di granturco" rappresentano l'eccezione più che la norma.
} 
L'autore di Fontamara, di fronte al "tentativo di contrapporre polemicamente al vecchio stato d'animo di angoscia esistenziale un atteggiamento di fiducia nel mondo e negli uomini” (PETRONIO apud ESPOSITO, 1987), mantenne un atteggiamento fatto di "poche certezze intrise di amaro pessimismo, e tormentato da un'ansia religiosa, sia pure di carattere più immanentistico che trascendente, che fu del tutto estranea alla natura e alle finalità del neorealismo" (ESPOSITO, 1998, p. 8184). Quello di Silone è stato definito realismo cristiano più che realismo sociale, o realismo esistenziale (PAMPALONI, 1971) o allegorico.

Come sottolinea Cesare Segre, Silone, in definitiva, rifugge dal gergo plebeo e dai toni volgari non meno che dalla parola aulica. L'esperienza narrativa dello scrittore s'inserisce nella "linea maestra" del romanzo post-manzoniano della "lingua media" aperta a inflessioni dialettali nella caratterizzazione dei personaggi. Come in Verga il popolo non viene osservato con distacco, ma diventa protagonista e gli avvenimenti sono narrati attraverso il filtro di una sensibilità collettiva. In particolare, il noi usato dai protagonisti traduce una modalità di presenza collettiva all'azione rappresentando un'estensione dell'io narrante demandato dall'autore non solo a Giovanni, Maddalena e al figlio dei due, ma a tutti gli uomini e le donne che i tre richiamano nella descrizione diretta degli avvenimenti, o nei commenti e nelle riflessioni prodotte dagli avvenimenti stessi, come "naturale proiezione comune della coscienza della loro identità e omogeneità di classe e di organismo sociale" (SEGRE, 1963, p. 7-8).

Già nel secondo capitolo l'uso marcato del noi rappresenta il punto di approdo di un viaggio che ha spinto le donne oltre l'egoismo e l'isolamento individuale che caratterizzava i personaggi nelle prime pagine del libro, un viaggio destinato a precedere quello che compiranno Giovanni, Maddalena e il loro figlio per giungere al cospetto dello scrittore. E il resoconto dei tre, a sua volta, risulterà un'estensione di quanto già testimoniato con le cinquecento copie del Che fare?, il primo passo verso un'ulteriore realizzazione del loro rapporto conquistato, per dirlo rilkianamente, nella forma scritta del romanzo. E la prefazione dell'autore, attraverso l'uso del pronome personale noi, si pone come un finale ideale a testimonianza di una raggiunta coscienza di sé in grado di rappresentare ogni cafone.

Il giornale implica un possibile, e diversamente inimmaginabile, contatto con i cittadini, in uno spazio che si fa comune. Esso non è solo un mezzo o uno stile ("un giornale di cafoni, il primo giornale dei cafoni. Un giornale scritto a mano.” p. 193), ma esprime la nuova volontà di essere direttamente in una lingua, diversa da quella propria, senza che intervenga più il senso estraniante della traduzione.

Questa operazione non cancella del tutto i limiti preesistenti giacché, come scrive Francesco Di Gregorio, l’autore “dentro il ‘bisogno’ di rivolgersi a tutti matura 
l'esigenza di esprimersi nella lingua di tutti, cioè nella lingua 'convenzionale' che, proprio per essere di tutti, non è, ontologicamente, di nessuno” (1991, p. 11). Tuttavia, come fa notare Doris Cavallari, lo scrittore, traducendo in italiano, in una lingua in prestito, ma raccontando i fatti nella maniera fontamarese, dà vita al dialogismo testuale e pertanto

ao transportar a realidade do dialeto para o italiano, o autor 'assume a morte do que foi oral, mas insere no seu texto outros contextos' [RUI, 1979]. O 'emprestimo' da língua do autor possibilita uma abrangência maior de interlocutores, de ouvintes/leitores dessas reflexões, porque a tradução da voz dialetal facilita a inserção de uma realidade local e fechada em un universo muito mais amplo. (2001, p. 36)

Ed è proprio rendendosi ibrida per mezzo della traduzione e della sua mediazione che la lingua del cafone riesce a mostrarsi e a rendersi osservabile dall'esterno, realizzando le proprie qualità specifiche. La lingua nazionale, come veste, riesce ad esaltare le forme di un dialetto il quale, se mostrato nella sua nudità, non avrebbe alcuna eloquenza. Il registro stilistico dell'opera riflette allora quello che è il costante sforzo dell'autore, come dei personaggi, di resistere alla tentazione di un dolce oblio nella propria memoria e nella propria lingua, libera di essere se stessa, ma in una dimensione chiusa e quasi inconsapevole, per proiettarsi verso un doloroso spazio di salvezza, anche per la lingua stessa, che pure è altra cosa, altro luogo.

Il Che fare? è la prova che i Fontamaresi, con le loro cinquecento copie distribuite appena prima dell'arrivo delle milizie fasciste, quasi dei messaggi in una bottiglia, sono giunti al possesso di una parola scritta. Attraverso la scrittura i personaggi di Fontamara scoprono di volersi e di potersi spingere oltre la contingenza e la finitudine del linguaggio che hanno sempre utilizzato. Il Che fare?, che è già un dire prima che un voler fare, diventa segno e testo portatore di significato. La domanda-titolo del giornale, posta al termine della narrazione, pur proiettando lo sguardo del lettore in avanti, rappresenta la chiusura di un cerchio: se tante vicissitudini hanno portato alla parola, ora la parola guiderà l'azione e l'interrogazione è il sigillo posto al termine del cammino fatto fin lì dai cafoni. Ciò che sulle prime ha le sembianze di una domanda retorica, fatalmente votata alla rassegnazione, appare in ultimo come un'insegna capace di indicare molteplici direzioni. E improvvisamente le loro esistenze si dischiudono in virtù di un finale incompiuto dove l'apparente mancanza di speranza è segno dell'apertura di un nuovo corso, non necessariamente armonioso, ma certamente diverso dal ciclo stagnante che li ha oppressi fino ad allora. In questo senso, un'importanza centrale riveste il personaggio di Berardo Viola. 


\section{Berardo}

Il sacrificio del giovane Fontamarese assume il senso di un avvento, di qualcosa che stabilisce, pseudo cristianamente, un prima e un dopo. Il ragazzo è l'incarnazione di una figura mediatrice capace di guidare tutti i suoi simili verso un'insperata dignità.

È dalla precarietà del mondo e dalla debolezza dell'uomo che l'autore inizia la sua parabola. Silone rivolge infatti il suo sguardo, oltre che sulla volgarità del fascismo (della quale dà un saggio emblematico attraverso l'episodio dello stupro), in special modo sulle connivenze che consentirono al fascismo di diffondersi. Attraverso figure come quelle di Carlo Magna, il canonico Don Abbacchio o gli ignavi carabinieri, l'autore ci presenta, oltre al male, il terreno fertile nel quale il male è riuscito ad attecchire, e sembra ricordare che il fascismo, per quanto costruito sulla violenza e sull'iniquità, seppe emergere in un contesto di democratizzazione. L'impresario in fin dei conti non è che un imprenditore per il quale la politica non è che una perdita di tempo, un sindaco assenteista che si è fatto eleggere unicamente per annullare la sua carica. E al contempo svela la latente affezione dei cafoni per quel mondo trascorso che li opprimeva e li accudiva e nel quale vigeva un "ordine irremovibile" imperniato sulla "legge dei preti, dei padroni e dell'abitudine” (p. 78). Il personaggio di Michele Zompa è esplicito a riguardo quando dice che un governo composto da un solo grande ladro mangia sempre meno di un governo di cinquecento ladri piccoli e affamati (p. 93). Tra la loro posizione e quella dell'Impresario i contadini riconoscono l'esistenza di figure, per così dire, intermedie come quelle di Carlo Magna, spolpato dai vizi e dalle banche del nuovo governo, o del fantasma del vecchio barone decaduto tanto caro a Baldissera. Sono figure alle quali i Fontamaresi non riescono ad opporre un vero rifiuto e verso le quali non mostrano mai un'aperta ostilità (lo stesso Don Circostanza sarà chiamato carogna solo alla fine del romanzo), ed è questo che li rende alieni da qualunque proposito rivoluzionario, o che li renderebbe inadeguati in un immediato quadro d'azione. Ciò, pertanto, non può condurli che verso una rivalsa spinta ben oltre il semplice riscatto politico e mondano, il cui protagonista è Berardo Viola.

La natura del giovane colloca la narrazione in una dimensione più religiosa o pseudo religiosa che prettamente ideologica. Nipote di un brigante morto impiccato, sua madre parla di lui ripetutamente, e in modo piuttosto lugubre, come di un condannato a morte dal destino ineluttabile. Nelle pagine che lo riguardano Silone ha modo di dipingerlo dapprima come un individuo generoso ma aggressivo, con tratti talora violenti per un impulsivo e passionale desiderio di giustizia, ed in seguito come un comune contadino remissivo disposto a piegarsi alle angherie dell'Impresario per amore della sua Elvira. 
La storia tra i due ragazzi è solo accennata, una scelta che, innanzitutto, pone in risalto l'immenso pudore su cui è fondato il loro amore e l'universo dei cafoni. La convenzionalità della loro vicenda ha il compito di delineare i tratti, quanto mai umani, di due futuri martiri. Nel caso di Berardo, vediamo il giovane esitare, perdere la speranza o peccare di egoismo fino agli ultimi istanti che precedono la sua scelta tragica. Non ci è dato di sapere con certezza se il ragazzo muoia davvero suicida o per i maltrattamenti subiti, ma ciò lo avvicina ancor di più alla figura di Cristo, al quale, comunque sia, viene esplicitamente paragonato (p. 185). Infatti, se da una parte la sua morte è il risultato di una flagellazione inflittagli dagli altri, l'ipotesi del suicidio, e più in generale la scelta di portare su di sé tutto il peso di una colpa non commessa, ce lo fa accostare a Gesù il quale accoglie e, sotto taluni aspetti, cerca la propria fine. E come Gesù verrà rinnegato, al termine del penultimo capitolo, dal figlio di Giovanni e Maddalena, suo compagno di cella.

Silone non reifica la figura di Cristo confinandola nello spazio troppo angusto del socialismo, ma la estende a una condizione umana degradata che ormai Stato e Chiesa hanno dimenticato. In questo modo anche i militanti improvvisati come Berardo, per il quale la rivoluzione dell'Avezzanese rappresenta solo un'occasione, riscoprono e riaccolgono pienamente il verbo cristiano nel proprio seno, oltre ogni consapevolezza, e dunque in modo ancora più sublime. Così facendo si rigenera la troppo arida $\mathrm{e}$ sclerotizzata figura del Cristo Socialista, estendendone il portato filosofico e l'anelito spirituale al di là dello spazio angusto della sua lettura ideologica, riorientandola e realizzandola.

Lo scrittore apre una terza via tra il polo della religione codificata e quello dell'ortodossia marxista, "qualcosa di nuovo" (p. 187) davanti al quale non gli riesce di aggiungere altro o non è necessario aggiungere altro, come accade a Berardo nel proferire le ultime parole. Oltre questa scelta esiste la possibilità di una testimonianza solo nel silenzio del proprio corpo votato al martirio, riaccostandosi così alla condizione primigenia dei cafoni incapaci di parola, o di una parola compiuta. Tutto si raccoglie in lui e diventa testimonianza realizzata con la propria carne che nei cafoni si farà parola, parola scritta.

La scelta finale supera sia l'eros che la politica, affinché il ragazzo possa darsi agli altri, "il primo cafone che non muore per sé, ma per gli altri" (p. 187), la sua grande scoperta definitiva. Questa illuminazione ha l'effetto di dare al suo destino un'impronta nuova e non più fatalmente tetra, e lo stesso amore per Elvira, vissuto sempre come in una clausura, e la morte della ragazza, diventano per lui una spinta ad andare oltre, perché il suo destino si compia. Persino la madre, dopo la sua morte, sembra comprendere la portata di un tale sacrificio: 
«Pur di riuscire, egli era veramente disposto a tutto. Per amore di una donna. La morte della donna forse lo ha salvato.»

«Strana salvezza morire in carcere» ripeté l’altra tra le labbra. [...]

«Nessuno può sapere» continuò la madre con voce di collera. «Forse la salvezza di Berardo è stata di essere restituito al suo destino.» (p. 191)

L’apparente marginalità con cui viene resa nota la morte di Elvira a Berardo e al lettore, e la maniera succinta che ha di narrarla Maria Grazia nell'ultimo capitolo, non hanno nulla di sommario. La figura della ragazza è inscindibile da quella di Berardo. I due si ritroveranno solo nella morte, nella misura in cui in vita si sono cercati, voluti, ma mai toccati. La ragazza, oltre che partecipe dello stesso destino del suo uomo, è parte di una medesima identità. Il personaggio può apparire privo di sottigliezze psicologiche, ma solo perché destinato anch'esso ad eludere, come Berardo, la trama degli eventi ordinari. La morte fulminea ed inspiegabile, per un'improvvisa febbre, appare come una repentina ascensione non mediata da eventi terreni. Come scrive Carmelo Aliberti:

Il personaggio di Elvira, in cui risultano sintetizzati i lineamenti di tante figure femminili della nostra letteratura, e in cui l'immagine della stilnovistica donna angelicata sembra fondersi con la rassegnata disposizione al sacrificio della Lucia manzoniana e con la pietrificata sofferenza delle creature verghiane, evidenzia un inedito spessore di protagonista, svolgendo un ruolo di riscontro dialettico e di proposta positiva alle dissacranti accuse di Silone. Con l'offerta della sua vita per la salvezza di Berardo, Elvira esprime la profondità del sentimento religioso nell'anima contadina innalzata così a vera depositaria del cristianesimo. (1977, p. 39-41).

La rapidità con cui si risolve il destino di Elvira è il segno dell'immediatezza di una volontà divina adempiuta.

Le altre figure femminili hanno senza dubbio molto colore, ma appaiono grottesche in più di un occasione e, se non vengono parodiate dallo scrittore, sembrano riflettere la parte più prosaica e talora goliardica dell'animo femminile. Eppure, è proprio questo il tratto più felice di Fontamara.

\section{Fontamaresi}

Se è vero che sonole figure di Berardo ed Elvira ad innalzarsi a depositarie diun pensiero forte e di un'umanità “alta” è altresì vero, come sostiene Claudio Varese, che l’autore 
raggiunge meglio una sua forza rappresentativa quando entra nel mondo e nel linguaggio dei cafoni, li riassume e li accoglie; l'ironia, il grottesco, la beffa, sono tra le forme più felici, ispirate e riprese direttamente, molto spesso, dalla fantasia, dai miti e dalla vita dei paesani della Marsica, e ne continuano e approfondiscono il senso amaro e duro della realtà. (1967, p. 151)

Quando Silone si adegua al modo dei cafoni, continua Varese, e ne ripete con segno intellettuale l'ironia attraverso cui si difendono dalla retorica ingannevole delle istituzioni, egli trova le sue parole più giuste, mentre nelle evasioni e sopraffazioni mistiche, sentimentali e moralistiche manca di esperienza letteraria e non può rifarsi al suo mondo dialettale.

L'ironia e la polemica sociale vengono a maturarsi dentro lo stesso mondo primitivo. La costruzione intellettuale dell'autore riprende, dalla fantasia locale e dal folklore, delle forme ingenue e sapienti di deformazione, che coincidono con atteggiamenti espressionistici. (1967, p. 151).

Se ne ha un esempio nell'episodio in cui i Fontamaresi sono chiamati ad acclamare, senza alcuna facoltà di comprensione dell'evento, il passaggio del Prefetto nella sua automobile lucidissima, portandosi appresso lo stendardo di San Rocco al posto del gagliardetto, episodio che il Varese definisce degna della matita del Grosz. E vale la pena citare nuovamente la partenza delle donne per il capoluogo nel secondo capitolo, un passaggio nel quale l'ironia, solo apparentemente bozzettistica, rivela le forme pregnanti di un universo corale.

A dispetto delle letture verticali possibili, ciò che rimane, ed è più facile trattenere dei personaggi, è l'efficacia e la densità dei loro detti popolari, come "Chi ha mangiato la pecora adesso vomita la lana" (p. 49) o "La morte dell'asino se la piange il padrone" (p. 54), o delle metafore del tipo "Albero spesso trapiantato, mai di frutti è carico" (p. 82) o di immagini come quelle di una Donna Clorinda che tiene due candele accese di fronte alla statua di Sant’Antonio perché venga “un'accidente” all’Impresario (p. 155). Detti, metafore o immagini che hanno la funzione di riflettere la realtà più intima di un mondo subalterno, quanto mai coeso nella sua varietà, e che in definitiva si estende ben al di là del semplice borgo di Fontamara.

È la teatralità delle forme dell'espressione, che fonde codici ideologici e iconologici arcaici e primitivi, come scrive Pietro Spezzani, ad essere "la forma artistica più rilevante del messaggio narrativo di Fontamara, a cui il contenuto popolare della narrazione fornisce un supporto chiaramente riconoscibile” (1979, p. 83). Per certi aspetti, Berardo 
ed Elvira sono gli unici personaggi seri del romanzo, per quanto non manchino certo i momenti drammatici. Il cuore di Fontamara è nelle facce e nelle espressioni dei protagonisti, a stretto contatto con la superficie del mondo. È qui che cielo e terra s'incontrano e s'incarnano non più in un solo uomo (Berardo), ma in un'intera comunità, ed è sempre qui che la voce narrante spesso si "pluralizza oggettivamente in un'esigenza di assolutizzazione” (ALIBERTI, 1977, p. 26).

Questo tratto stilistico ha le sue proficue ricadute sull'aspetto religioso dell'opera, mitigandone così le pesantezze. I cafoni, infatti,

hanno qualcosa di tragico e di profondo e, nella loro ignoranza, vedono e sanno più delle persone istruite; parlano con immagini, con grandiose allegorie. Questo è il punto più sicuro, più forte e a un tempo più personale dell'artista Silone e vi coincidono diversi atteggiamenti, anche apparentemente contrastanti, ingenui e insieme ingegnosi, frutto di una ignoranza eppure facilmente riconducibili ad una cultura consapevole e raffinata. (VARESE, 1967, p. 155)

Per quanto somigli “ad ogni villaggio meridionale che sia un po' fuori mano” (p. 7), Fontamara è un'eccezione universale, un caso unico nel circondario, lo dimostra la fama che il borgo ha assunto agli occhi dei cittadini che si burlano continuamente dei suoi abitanti. E le maschere grottesche, deformi ed improbabili di quest'ultimi, più che la forza della verità, sono ciò che salvano, pur dissimulandolo, un dolore che nella storia è destinato, di norma, ad andare perduto, e che saldano alla denuncia di un male assoluto e senza tempo il volto, oltre che l'essenza, di quegli uomini che dal male furono cancellati.

\section{Silone}

La contraddizione, presente in Silone, tra le premesse cristiane e gli esiti morali delle sue opere ha spinto la critica a considerare lo scrittore ora come un autore cristiano, altre volte come un eretico, o un apostata o addirittura un non cristiano. Come scrive Vittoriano Esposito, egli

non si è mai posto in termini chiari il problema del destino ultimo dell'uomo dopo la morte, né quello della fine del mondo. Pochi giorni prima della morte, egli dichiarò di credere in Dio, ma non nell'aldilà: affermazione che esclude proiezioni ultraterrene della sua visione religiosa e la tiene strettamente legata al mondo umano, che per lui resta il solo mondo possibile. (1998, p. 85) 
Lo spazio umano sulla terra è l'unico mondo possibile ed è il mondo di una sola volta e di una sola occasione, se si pensa a quanto dice Berardo nei suoi ultimi istanti di vita. ${ }^{4}$ Come per Rilke, vi è solo un aldiqua nel quale agire e in questo spazio è il passare e il ripassare dell'uomo sulla (propria) terra e il portarsi oltre tutti gli eventi che fa maturare, nel cuore dell'uomo, il tempo della parola o, nel caso di Berardo, la possibilità di approdare ad una morte fecondatrice di parola che non sia al di là delle proprie forze. Giovanni, riferendosi al giovane Fontamarese, dice:

Come può un uomo della terra rassegnarsi alla perdita della terra? Quella terra era già stata del padre di Berardo, e Berardo vi aveva anche lui faticato dall'età di dieci anni. Fra la terra e il contadino, dalle nostre parti, ma forse anche altrove, è una storia dura e seria, è come tra marito e moglie. È una specie di sacramento. Non basta comprarla, perché una terra sia tua. Diventa tua con gli anni, con la fatica, col sudore, con le lagrime, con i sospiri. (p. 68).

Sono parole che sembrano rimandare, sia pure in maniera labile, prosaica, senza alcuna tensione mistica, all'universo della poesia rilkiana.

Perché, dunque, la centralità della parola? Ai contadini viene a mancare un passato (gli antichi retaggi) e, nel momento in cui l'acqua viene loro negata, anche un futuro; non hanno niente dietro di sé e niente davanti e Berardo a un certo punto non è più vincolato dall'amore di Elvira. Nel loro eroismo, inteso come l'atto di spingersi, loro malgrado, oltre ogni evento e oltre se stessi, la parola scaturisce come l'unico esito possibile. Di fronte al fallimento degli antichi signori (Carlo Magna e il Barone), all’Impresario che spazza via anche i tratturi, ${ }^{5}$ al Fascismo, cui non si oppone alcuna reazione violenta, alla latitanza di una Chiesa pavida o corrotta e alla mancanza di una coscienza dell'identità nazionale (anche da parte loro), o ridotta, al più, a una patacca d'argento su uno zinale ${ }^{6}$ i cafoni, come un vaso colmo che sta per traboccare, una volta saputo della morte di Berardo, riversano le loro parole su un giornale scritto a mano, decidendo, immediatamente e in modo naturale, di depositare anche loro la propria confessione e di diramare le cinquecento copie del Che fare? La loro incompiutezza rappresenta, sul piano della narrazione, una (ri)trovata libertà e la possibilità di realizzare nuovi percorsi come, liberamente, il loro destino.

La parola che l'intera comunità fontamarese ha intenzione di raggiungere è certamente una parola di verità, che possa raccontare la verità sui fatti di Fontamara. Di qui, però, un'ulteriore, feconda, contraddizione. Perché se è vero che la forza conclusiva dei cafoni sta nel sapersi riconoscere con l'altro in un medesimo destino, e si intendano per l'altro, ad esempio, i cafoni in rivolta ad Avezzano, dei quali si fa solo un rapido accenno

4. "Se io tradisco passeranno ancora centinaia di anni prima che una simile occasione si ripresenti" (p. 187).

5. Percorsi montani usati per la transumanza attraverso i quali si spostavano le greggi e che potevano estendersi fino ad altre regioni, in particolare la Puglia. Nel romanzo assurgono quasi a simbolo dell'arcaica condizione dei contadini, data la loro origine risalente a un tempo anteriore alla nascita di Cristo.

6. Il riferimento è alla medaglia avuta per il marito morto in guerra di cui Marietta fa sfoggio accingendosi a partire per il capoluogo con le altre donne (p. 35). 
al termine del libro, è anche vero che per poter intendere ed intendersi essi devono dare voce essenzialmente alla loro finitudine e al loro angolo di mondo, sia pur tanto misero e prossimo alla fine (o, anzi, proprio in forza di questo). È l'esistenza di uomini e donne nelle loro fattezze unici ed inimitabili a giustificare la nascita della parola. Di qui il valore preminentemente estetico dell'opera.

Francesco Di Gregorio, richiamando l'immagine della rosa che si compone come descritta da Silone al termine della prefazione, ${ }^{7}$ sostiene che il cafone "è nel dialettogergo della sua terra come le varie strutture della rosa sono nelle viscere della rosa" e che si debba rivolgere la propria attenzione "all'essenza interna del dato medesimo, alla ricerca di ciò che è 'dentro' il simbolo e che il simbolo, per sua costituzione, nasconde" (1991, p. 13).

Anche per questo, dunque, la verità avrà come suo emblema infine una domanda:

Michele propose un buon titolo: La verità, che voleva dir molto.

Ma Scarpone arricciò il naso: «La verità?» disse. «Chi conosce la verità?»

«Non la conosciamo, ma vogliamo conoscerla» rispose Michele.

«E quando l'avrai conosciuta», gli rispose Scarpone «con la verità ci farai il brodo?».

(p. 192)

Il fatto che La Verità e La Giustizia, altro titolo forte, ma troppo imparentato con i Carabinieri a dire di Scarpone, siano scartati in favore del Che fare? pare non essere un segno casuale.

Nel tracciare la traiettoria estetica dei suoi personaggi l'autore non va alla ricerca di una causa prima o di un fine ultimo, ma al contempo non lascia neppure che i suoi personaggi, nella loro originalità, appaiano dei mostri incapaci di metamorfosi. Il Che fare? sarà un giornale di cafoni attraverso cui comunicare con altri cafoni, ma scritto in italiano, un italiano nel quale si sforzano di esprimersi con un nuovo vigore e un'insolita partecipazione, dato lo scopo comunicativo, e dunque offerto, in ultimo, a chiunque voglia leggerlo. La scrittura e la comunicazione li spingono sempre di più tra le braccia di un nuovo linguaggio che per loro è vita, una lingua nuova, non più mera interprete, capace di accogliere anche la loro consueta comicità involontaria se Marietta, non sapendo con quante elle si debba scrivere il cognome Viola, dice di poterlo fare in modo che rimanga dubbio se ve ne siano una o due. Improvvisamente, e in modo assai precario, si scoprono cantori di una realtà in probabile estinzione mentre vanno anche loro nel doppio regno, dove morendo portano con sé la vita. 


\section{Bibliografia}

ALIBERTI, C. Come leggere Fontamara di Ignazio Silone. Milano: Mursia, 1977.

CAVALLARI, D.N. A tessitura narrativa de Fontamara e Vidas secas. Insieme, São Paulo, n. 8, pp. 34-45, 2001.

DI GREGORIO, F. Silone e le ragioni del “bello scrivere”. In: Letture siloniane, Fontamara, vol. I. L’Aquila: Amministrazione provinciale, 1991.

ESPOSITO, V. Silone e il Cristianesimo. In: Silone vent’anni dopo (Ricognizioni e prospettive critiche). L’Aquila: Amministrazione Provinciale, 1998, pp. 85-91.

PAMPALONI, G. Realismo esistenziale. Roma: De Luca, 1971.

PETRONIO, G. L'attività letteraria in Italia. Palermo: Palumbo, 1987.

RUI, M. Entre mim e o nómada: a flor. África, Literatura, Arte e Cultura, 1979, pp. 541-543.

SCARAMUCCI, I. Silone e la letteratura. In: BRAMBILLA, R. (Org.). I. Silone tra testimonianza e utopia. Atti del seminario di studio (Assisi-ottobre 1979). Assisi: Biblioteca della Pro Civitate Christiana, 1980.

SEGRE, C. Lingua, stile e società. Milano: Feltrinelli, 1963.

SILONE, I. Fontamara. Milano: Mondatori, 1998.

SILONE, I. Uscita di sicurezza. Milano: Mondadori, 1980.

SPEZZANI, P. Fontamara di Silone: grammatica e retorica del discorso popolare. Padova: Liviana, 1979. VARESE, C. Occasioni e valori della letteratura contemporanea. Bologna: Cappelli, 1967. 